\title{
Wasserstoffmessung in Prozessgasen
}

\author{
U. Sasum ${ }^{1}$, U. Guth ${ }^{2}$ \\ ${ }^{1}$ Forschungszentrum Sensorik Greifswald e.V., Brandteichstraße 19, 17489 Greifswald / D \\ sasum@fsg-ev.de \\ ${ }^{2}$ TU Dresden, FB Chemie und Lebensmittelchemie, 01062 Dresden / D
}

\begin{abstract}
Kurzfassung:
Im Beitrag werden Untersuchungen zur Langzeitstabilität von Sensorkombinationen zur Wasserstoffmessung in Transformatorgasen, die in Gasmonitoren eines kommerziellen Anbieters über einen Zeitraum von bis zu zehn Jahren betrieben wurden, vorgestellt. Diese Ergebnisse werden mit denen eines Wasserstoffsensors, der die Änderung der optischen Eigenschaften einer Palladiumclusterschicht beim Kontakt mit Wasserstoff zur Messung ausnutzt, hinsichtlich Stabilität und Querempfindlichkeiten zu typischen Transformatorfehlergasen verglichen. Die Änderung der Wasserstoffsensitivität wurde in einem Laborteststand über mehrere Monate verfolgt.
\end{abstract}

Key words: Wasserstoff, Transformatorgas, Onlinemessung, Langzeitstabilität, Sensoren

\section{Einleitung}

Die Analyse gelöster und freier Gase (Dissolved Gas Analysis - DGA) von in Betrieb befindlichen, mit Mineralöl impregnierten elektrischen Betriebsmittel ist eines der am häufigsten verwendeten diagnostischen Hilfsmittel zur Erkennung und Bewertung von Fehlern in elektrischen Geräten [1]. Weltweit werden jedes Jahr tausende DGA in Laboratorien durchgeführt. Die dafür geeigneten Verfahren und Geräte sind in Normen (z.B. DIN EN 60567 [2]) beschrieben. Als Analysegeräte werden generell Gaschromatographen genutzt.

In den letzten 20 Jahren sind Online-Geräte mit dem Ziel entwickelt worden, kontinuierlich zu messen und Probenahmefehler auszuschließen. Eine CIGRE-Arbeitsgruppe hat 13 auf dem Markt befindliche Online-DGAMonitore hinsichtlich ihrer Genauigkeit im Vergleich zu Laboranalysen untersucht. Diese Geräte unterschieden sich hinsichtlich der genutzten Gasextraktionsverfahren (Vakuumextraktion, Membrantechnik, Gleichgewichtsverfahren) und der analytischen Messverfahren. Neben Online-Gaschromatographen kommen Geräte zum Einsatz, die z.B. mit IRSpektrometern, NDIR-Sensoren, photoakustischen oder elektrochemischen Sensoren sowie Kombinationen daraus arbeiten [3]. Die Messung von Wasserstoff als universellem Transformator-Fehlergas ist die wichtigste Aufgabe aller Online-Messgeräte. Einfache Überwachungseinheiten sollen nur Änderungen der Wasserstoffkonzentration im Öl detektieren.
Es gibt eine Reihe auf dem Markt bzw. in Entwicklung befindlicher Sensoren für die Wasserstoffmessung, die auf sehr unterschiedlichen Messprinzipien beruhen [4]. Die Fortschritte im Bereich der Nanotechnologie beförderten die Entwicklung von Wasserstoffsensoren auf Basis von Palladium-Nanostrukturen [5].

Der in Onlinegeräten einzusetzende Sensor bzw. das Sensorsystem hat eine Vielzahl von Anforderungen zu erfüllen: hohe Selektivität für Wasserstoff, hohe Sensitivität, Reversibilität und Reproduzierbarkeit der Messeffekte, relativ schnelles Ansprechverhalten, Langzeitstabilität und niedrige Kosten in Produktion, Betrieb und Wartung.

Unter Langzeitstabilität versteht man in der Transformatorgasanalyse einen Zeitraum, der möglichst der Lebensdauer des zu überwachenden Transformators entspricht, mindestens aber mehrere Jahre umfasst. Es sind bisher keine Veröffentlichungen zur Langzeitstabilität von Online-DGA-Monitoren erschienen. Auch im CIGRE-Report wurden dazu keine Aussagen getroffen. Ein Wasserstoffsensor auf Basis von PalladiumNanostrukturen für den Einsatz in Transformatorüberwachungsgeräten wird in der Literatur beschrieben [6]. Veröffentlichungen zu Betriebserfahrungen mit diesem Sensor an Transformatoren gibt es bisher nicht.

\section{Gase in luftatmenden Transformatoren}

In luftatmenden Transformatoren steht das Öl über eine Trocknungsvorlage in Kontakt mit der 
Umgebungsluft. Die Luftbestandteile lösen sich im entgasten Öl bis das Verteilungsgleichgewicht eingestellt ist. Umgekehrt werden alle Verbindungen, die im Transformatoröl in höheren Konzentrationen als der entsprechenden Gleichgewichtskonzentration in der Umgebungsluft vorhanden sind, ausgeatmet. Wie stark die Abnahme der Konzentration im Öl ist, hängt von der Löslichkeit der Verbindung ab, die Geschwindigkeit von der Offenheit des Transformators, die von seiner Bauart und der Betriebsweise bestimmt wird. Im Öl gelöstes Wasser stammt überwiegend aus der Festisolation. Sowohl das Verteilungsgleichgewicht des Wassers zwischen Festisolation und Öl, als auch die Löslichkeit des Wassers im Öl ist stark temperaturabhängig. Wasserkonzentrationen unter $20 \mathrm{mg} / \mathrm{kg}$ werden hinsichtlich der Isolierölqualität für alle Transformatorklassen als mindestens ausreichend angesehen [8]. Bei höheren Konzentrationen können Ölaufbereitungsmaßnahmen notwendig sein.

Tab. 1: Gaskonzentrationen in Transformatoren

\begin{tabular}{|c|c|c|c|c|}
\hline \multirow{2}{*}{ Gas } & \multirow{2}{*}{$\begin{array}{c}\mathrm{k} \\
\text { bei }\end{array}$} & \multicolumn{3}{|c|}{ Gaskonzentration / ppm (v/v) } \\
\cline { 3 - 5 } & & $5^{\circ} \mathrm{C}$ & $\begin{array}{c}\text { in der } \\
\text { Luft [7] }\end{array}$ & \multicolumn{2}{|c|}{ gelöst im Öl } \\
\cline { 4 - 5 } & & & $\begin{array}{c}\text { aus der } \\
\text { Luft }\end{array}$ & $\begin{array}{c}\text { Fehler- } \\
\text { gase }\end{array}$ \\
\hline $\mathrm{Ar}$ & 0,15 & 9300 & 1395 & - \\
\hline $\mathrm{H}_{2}$ & 0,055 & 0,5 & $<1$ & $50-100$ \\
\hline $\mathrm{O}_{2}$ & 0,172 & 209500 & 36034 & - \\
\hline $\mathrm{N}_{2}$ & 0,091 & 780800 & 71052 & - \\
\hline $\mathrm{CO}^{n}$ & 0,132 & 0,2 & $<1$ & $400-600$ \\
\hline $\mathrm{CO}_{2}$ & 1,09 & 400 & 436 & $3800-$ \\
& & & & 14000 \\
\hline $\mathrm{CH}_{4}$ & 0,429 & 1,8 & $<1$ & $30-130$ \\
\hline $\mathrm{C}_{2} \mathrm{H}_{6}$ & 2,82 & $<0,1$ & $<1$ & $20-90$ \\
\hline $\mathrm{C}_{2} \mathrm{H}_{4}$ & 1,84 & $<0,1$ & $<1$ & $60-280$ \\
\hline $\mathrm{C}_{2} \mathrm{H}_{2}$ & 1,24 & $<0,1$ & $<1$ & $2-280$ \\
\hline $\mathrm{C}_{3} \mathrm{H}_{8}$ & 8,65 & $<0,1$ & $<1$ & k.A. \\
\hline $\mathrm{C}_{3} \mathrm{H}_{6}$ & 7,66 & $<0,1$ & $<1$ & k.A. \\
\hline
\end{tabular}

Die zur Berechnung der Gleichgewichtskonzentrationen in Tabelle 1 benutzten Ostwaldkoeffizienten $k$ entsprechen jeweils einem Mittelwert, der aus verschiedenen Transformator-Mineralölen bestimmt wurde [1], mit Ausnahme der Koeffizienten für $\mathrm{C}_{3} \mathrm{H}_{8}$ und $\mathrm{C}_{3} \mathrm{H}_{6}$, die aus [9] ermittelt wurden und des ArKoeffizienten (eigene, unveröffentlichte Ergebnisse für Shell Diala D). Sowohl durch die natürliche Alterung von Isolierstoffen als auch fehlerhaften Betrieb können sich Fehlergase im Transformator bilden und Sauerstoff verbraucht werden. Die in Tabelle 1 angegebenen Fehlergaskonzentrationen entsprechen den Bereichen der typischen $90 \%$-Werte, die weltweit aus 25 elektrischen Netzwerken mit über 20000 Leistungstransformatoren erhalten wurden [1].

\section{Messung von Transformatorgasen}

Bisher gibt es keine kommerziellen Sensoren, mit denen Gase direkt im Transformatoröl gemessen werden können. Die im Öl gelösten Gase müssen zur Messung in eine Gasphase überführt werden. Dabei resultieren abhängig von der Extraktionsmethode bei gleicher Konzentration im Öl verschiedene Gasraumkonzentrationen, wie in Tabelle 2 beispielhaft dargestellt ist.

Tab. 2: Abhängigkeit der Gasphasenkonzentration von den Volumenverhältnissen bei der Teilextraktion

\begin{tabular}{|c|c|c|c|c|}
\hline \multirow{2}{*}{ Gas } & \multicolumn{2}{|c|}{ Gaskonzentration / ppm (v/v) } \\
\cline { 2 - 5 } & im Öl & \multicolumn{3}{|c|}{ im Gas } \\
\cline { 2 - 5 } & & \multicolumn{3}{|c|}{$\begin{array}{c}\text { v(Gas) / v(ÖI) im } \\
\text { Extraktionsprozess }\end{array}$} \\
\cline { 2 - 5 } & & $1: 1$ & $1: 10$ & $<<$ \\
\hline $\mathrm{H}_{2}$ & 50 & 47 & 323 & 909 \\
\hline $\mathrm{H}_{2}$ & 1000 & 948 & 6452 & 18181 \\
\hline $\mathrm{O}_{2}$ & 32000 & 27304 & 117647 & 186046 \\
\hline $\mathrm{N}_{2}$ & 64000 & 58662 & 335079 & 703296 \\
\hline $\mathrm{CO}^{2}$ & 1000 & 883 & 4310 & 7576 \\
\hline $\mathrm{CO}_{2}$ & 20000 & 9569 & 16807 & 18349 \\
\hline $\mathrm{CH}_{4}$ & 500 & 350 & 945 & 1166 \\
\hline $\mathrm{C}_{2} \mathrm{H}_{6}$ & 500 & 131 & 171 & 177 \\
\hline $\mathrm{C}_{2} \mathrm{H}_{4}$ & 500 & 176 & 258 & 272 \\
\hline $\mathrm{C}_{2} \mathrm{H}_{2}$ & 500 & 223 & 373 & 403 \\
\hline $\mathrm{C}_{3} \mathrm{H}_{8}$ & 500 & 52 & 57 & 58 \\
\hline $\mathrm{C}_{3} \mathrm{H}_{6}$ & 500 & 58 & 64 & 65 \\
\hline
\end{tabular}

Die Bestimmung der Verfahrenskennwerte wie Messbereichsgrenze, Nachweisgrenze, Genauigkeit und Wiederholbarkeit muss für jedes DGA-System, egal ob Laborsystem oder 
Online-Gerät, für die Kombination aus Extraktionseinheit und eigentlichem Messgerät erfolgen.

\section{Online-Messungen von Wasserstoff mit dem Trafogasmonitor TGM}

Der Trafogasmonitor TGM der Gatron $\mathrm{GmbH}$ wurde 2002 in den Markt eingeführt. Mit den Geräten der Typen TGM-M 1.2 und TGM-M 1.3 konnten Wasserstoff, Sauerstoff, Stickstoff, Kohlenmonoxid und Kohlendioxid gelöst im Öl, der Gassättigungsgrad und die relative Ölfeuchte gemessen werden, außerdem Wasserstoff im Buchholzgas. Die Sensormodule enthielten einen speziell angepassten Wärmeleitfähigkeitssensor (WLD), einen paramagnetischen Sauerstoffsensor (PMA 1.11, M \& C) und NDIR-Sensoren zur Messung von Kohlenmonoxid bzw. Kohlendioxid. Diese Sensoren basieren auf den Typen SM-CO-P und SM- $\mathrm{CO}_{2}-\mathrm{P}$ der Ritschel Sensor $\mathrm{GmbH}$ und wurden für die Nutzung im TGMSensormodul optimiert. WLD und IR-Sensoren wurden unabhängig voneinander mit einer Regelgenauigkeit von 0,01K temperiert. Der Einfluss des Gesamtdrucks auf die Messung der IR-Sensoren wurde durch einen speziellen Messablauf im TGM-M eliminiert. Das Prinzip der Wasserstoffbestimmung beruht darauf, dass man vom WLD-Messwert, einem Summenparameter aus allen im Messgas enthaltenen Komponenten, die den zuvor ermittelten Konzentrationen von $\mathrm{O}_{2}, \mathrm{CO}, \mathrm{CO}_{2}$ und $\mathrm{H}_{2} \mathrm{O}$ adäquaten Spannungen subtrahiert und aus der Differenz die Wasserstoffkonzentration im Messgas berechnet. Die Angabe der Konzentrationen für $\mathrm{H}_{2}, \mathrm{~N}_{2}$, $\mathrm{O}_{2}$, $\mathrm{CO}$ und $\mathrm{CO}_{2}$ erfolgt als im Öl gelöstes Gas in $\mathrm{ppm}(\mathrm{v} / \mathrm{v})$.

\section{Langzeitstabilität der Sensormodule}

Im Zuge von Wartungsarbeiten konnten Sensormodule der Baujahre 2004 und 2005 erneut unter Kalibrierbedingungen gemessen werden. Allerdings wurden die Messungen nicht

Tab. 3: Langzeitstabilität des WLD 0041

\begin{tabular}{|c|c|c|c|c|}
\hline \multicolumn{5}{|c|}{ WLD 0041, ppm Gas / mV U WLD } \\
\hline & 2005 & 2006 & 2009 & 2011 \\
\hline $\mathrm{H}_{2}$ & 506 & 507 & 519 & 502 \\
\hline $\mathrm{O}_{2}$ & 26178 & 24876 & 26385 & 25240 \\
\hline $\mathrm{Ar}$ & -4312 & k.A. & -4417 & -4237 \\
\hline Methan & 2665 & k.A. & 2690 & 2443 \\
\hline
\end{tabular}

immer mit allen Gasen durchgeführt. In den Tabellen 3 und 4 sind WLD-Kennwerte zusammengefasst.

Tab. 4: Langzeitstabilität des WLD 0024

\begin{tabular}{|c|c|c|}
\hline \multicolumn{3}{|c|}{ WLD 0024, ppm Gas / $\mathrm{mV} \cup_{\text {WLD }}$} \\
\hline & 2004 & 2015 \\
\hline $\mathrm{H}_{2}$ & 487 & 481 \\
\hline $\mathrm{O}_{2}$ & 24302 & 23318 \\
\hline $\mathrm{Ar}$ & -4035 & -4267 \\
\hline
\end{tabular}

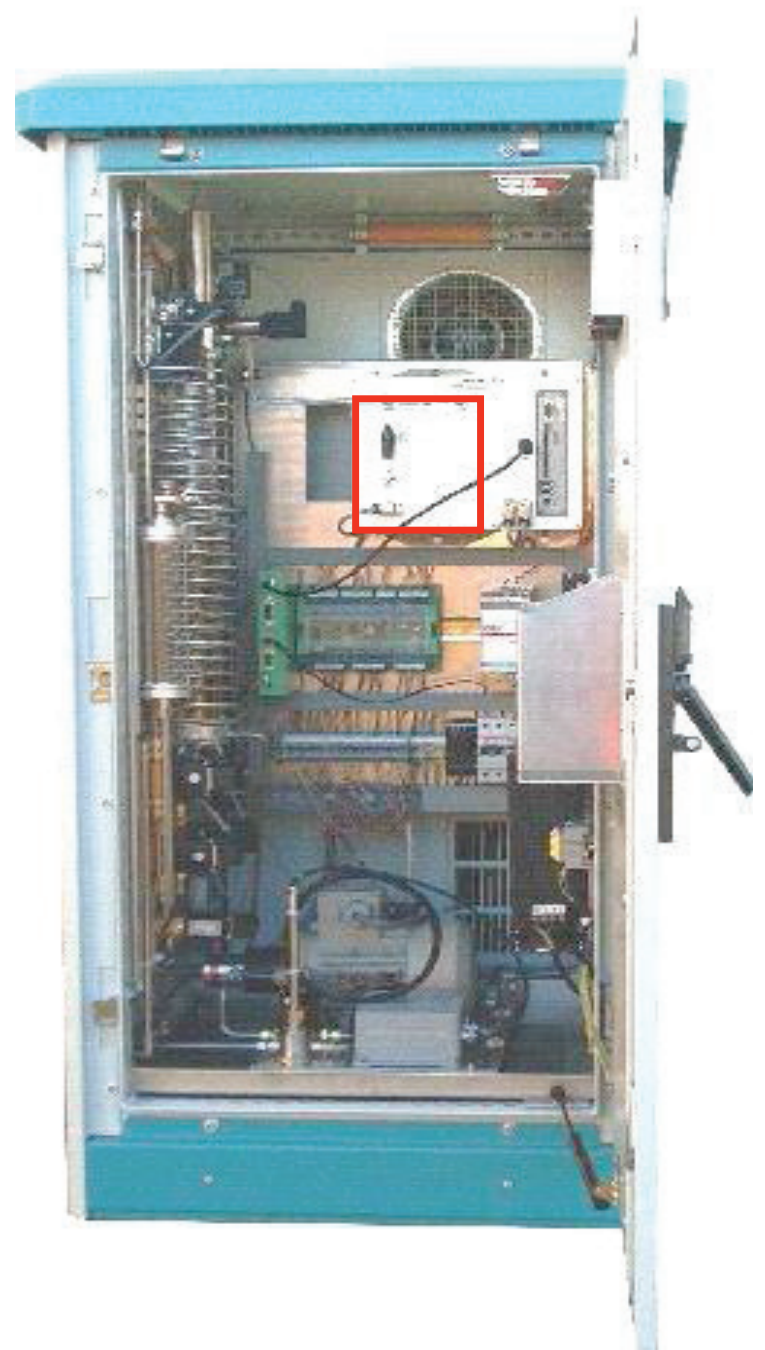

Bild 1 TGM-M 1.3, rot umrandet die Frontplatte des Sensormoduls

Es wird deutlich, dass keine systematischen Empfindlichkeitsänderungen der WLD während dieser Zeiträume aufgetreten sind. Die Abweichungen liegen innerhalb der Messunsicherheit des Verfahrens. Die 
Nachkalibrierungen weiterer WLD nach kürzeren Betriebszeiten bestätigten diese Ergebnisse.

Die Stabilität der Sensoren zur Messung von $\mathrm{O}_{2}$, $\mathrm{CO}$ und $\mathrm{CO}_{2}$ beeinflussen nicht nur die Richtigkeit der Konzentrationsangaben dieser Gase, sondern auch die des Wasserstoffs.

Im Bild 2 sind die Ergebnisse der Langzeitmessungen zweier $\mathrm{CO}_{2}$-Sensoren zusammengefasst. Um die Messgenauigkeit im

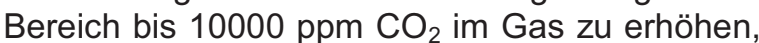
wurde u.a. eine Änderung der Küvettenlänge vorgenommen. Die Abweichungen der Langzeitvergleiche liegen innerhalb der Messgenauigkeit des Verfahrens.

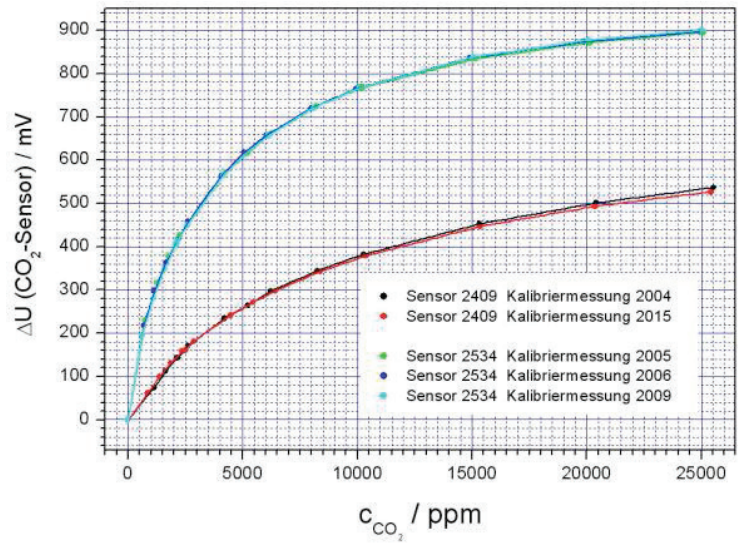

Bild 2 Langzeitstabilität von $\mathrm{CO}_{2}$-Sensoren

Für die Genauigkeit der Wasserstoffkonzentration ist aber nicht der relative Messfehler, sondern die absolute Abweichung der $\mathrm{CO}_{2}$-Konzentration vom richtigen Wert entscheidend. Die Empfindlichkeit des WLD gegenüber Kohlendioxid ist nicht linear. Sie nimmt mit größer werdender Konzentration zu. Die aus einem Messfehler des $\mathrm{CO}_{2}$-Sensors von $10 \mathrm{mV}$ resultierenden Fehler bezogen auf die Kohlendioxid- und Wasserstoffkonzentration der im Öl gelösten Gase sind in Tabelle 5 aufgezeigt.

Tab. 5: Einfluss des $\mathrm{CO}_{2}$-Messfehlers im Gas von $10 \mathrm{mV}$ auf die Konzentrationen im ÖI

\begin{tabular}{|c|c|c|c|c|}
\hline $\begin{array}{c}\mathrm{CO}_{2} \\
\text { Konzentration } \\
\text { im Messgas } \\
\mathrm{ppm}(\mathrm{v} / \mathrm{v})\end{array}$ & \multicolumn{3}{|c|}{$\begin{array}{c}\text { resultierender Fehler der } \\
\text { Gaskonzentration im Öl } \\
\text { gelöst / ppm (v/v) }\end{array}$} \\
\cline { 2 - 5 } & \multicolumn{3}{|c|}{ Sensor 2409 } & \multicolumn{2}{c|}{ Sensor 2534} \\
\cline { 2 - 5 } & $\mathrm{CO}_{2}$ & $\mathrm{H}_{2}$ & $\mathrm{CO}_{2}$ & $\mathrm{H}_{2}$ \\
\hline 1000 & 147 & $<-1$ & 65 & $<-1$ \\
\hline 5000 & 359 & -2 & 223 & $<-1$ \\
\hline 20000 & 1183 & -5 & 2240 & -9 \\
\hline
\end{tabular}

Wie im Bild 3 zu erkennen ist, unterlag auch der CO-Sensor keiner anhand der Messwerte sichtbaren Alterung. Die CO-Empfindlichkeit des WLD ist sehr gering, unterhalb von 1000 ppm vernachlässigbar. Selbst extreme Messfehler des CO im Gas bewirken nur geringe Fehler der Angabe der Wasserstoffkonzentration im Öl.

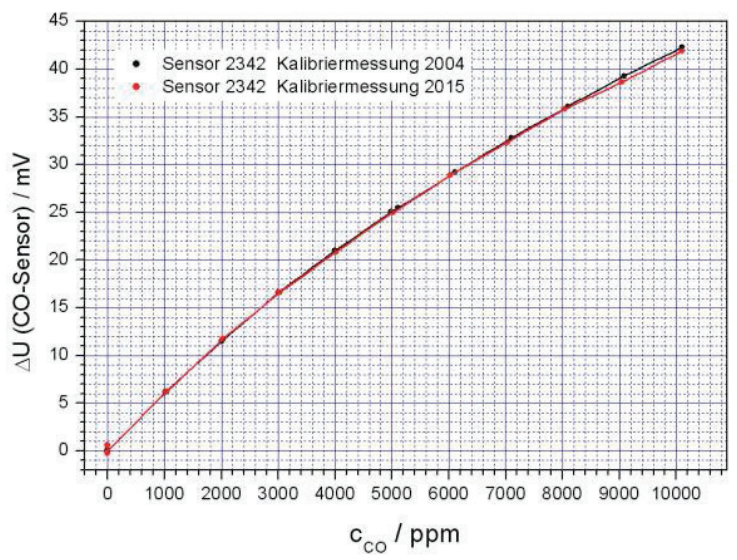

Bild 3 Langzeitstabilität eines CO-Sensors

Obwohl die IR-Sensoren temperiert wurden, war ein Einfluss der Umgebungstemperatur auf die Grundlinien der Sensoren zu beobachten, nicht aber auf deren Empfindlichkeit. Im TGM-M 1.3 wurden alle Sensoren regelmäßig mit trockener, $\mathrm{CO}_{2}$-freier Luft kalibriert. Die Gehäuseinnentemperatur des Geräts betrug im Winter ca. $27^{\circ} \mathrm{C}$. Im Sommer schwankte die Temperatur innerhalb der Schaltgrenzen des Kühlgerätes zwischen $32^{\circ} \mathrm{C}$ und $36^{\circ} \mathrm{C}$. Im Bild 4 ist die daraus resultierende jahreszeitliche Nullpunktschwankung des Sensors 2409 dargestellt.

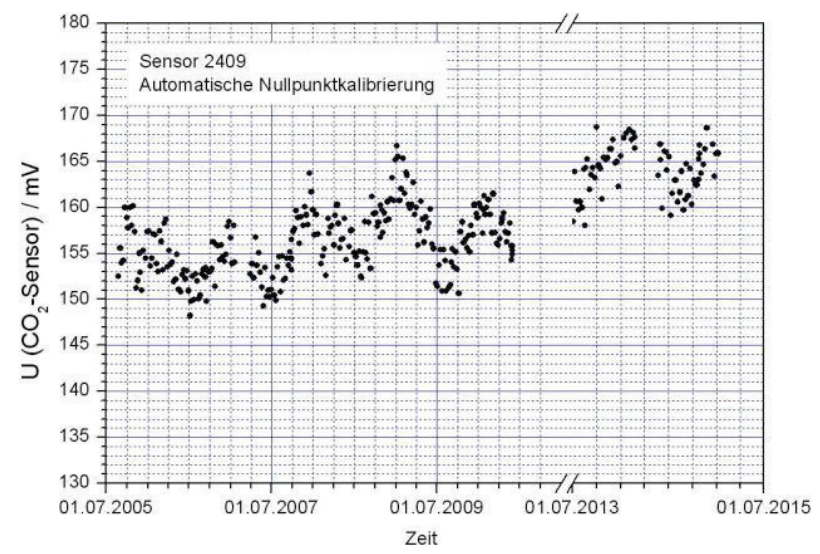

Bild 4 Automatische Nullpunktkalibrierung eines $\mathrm{CO}_{2}$-Sensors im TGM-M1.3

Die Sauerstoffkonzentration in luftatmenden Transformatoren kann über einen großen 
Bereich von $<1000 \mathrm{ppm}$ im Öl bis zur Gleichgewichtskonzentration variieren. Die Messung des Sauerstoffs mittels paramagnetischem Sensor wird durch die anderen, potenziell in Gasphasen über Transformatorölen enthaltenen Verbindungen nicht beeinflusst. Die Genauigkeit der Sauerstoffsensoren schwankte bei allen Untersuchungen nur im Rahmen der vom Hersteller angegeben Produktspezifikation. Analog zur Angabe in DGA-Laboruntersuchungsberichten werden Wasserstoffkonzentrationen unterhalb von $10 \mathrm{ppm}$ im TGM für den Kunden als <10 ppm angezeigt. In den Bilder 5 und 6 sind die an einem Umspannwerkstransformator über einen Zeitraum von mehr als 12 Monaten gemessenen originären TGM-Ergebnisse dargestellt.

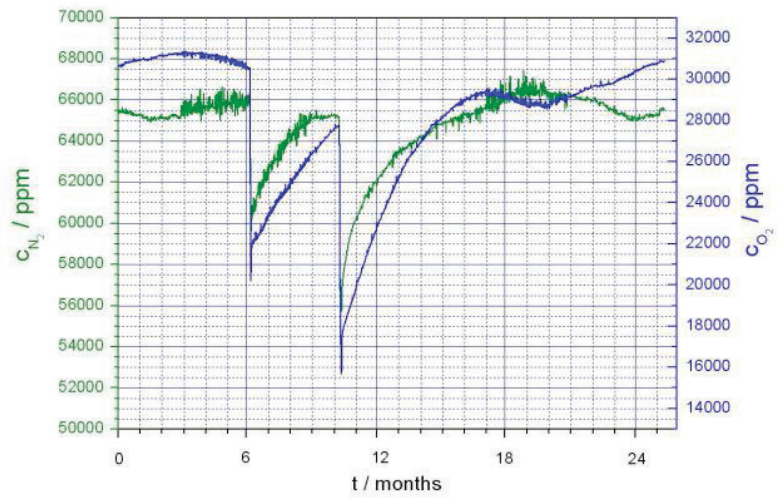

Bild 5: Onlinemessung von $\mathrm{O}_{2}$ und $\mathrm{N}_{2}$ an einem $110 \mathrm{kV}$-Transformator

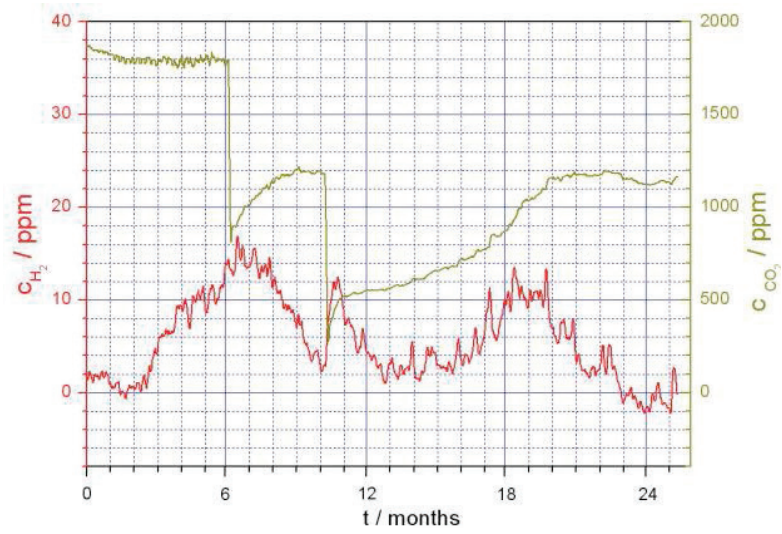

Bild 6: Onlinemessung von $\mathrm{H}_{2}$ und $\mathrm{CO}_{2}$ an einem $110 \mathrm{kV}$-Transformator

Im Überwachungszeitraum wurden zwei Ölaufbereitungsmaßnahmen (Entgasungen) am Transformator durchgeführt. Dadurch wurden Stickstoff, Sauerstoff und Kohlendioxid teilweise aus dem Öl entfernt. Bereits vor der ersten Entgasung konnte durch eine Laboranalyse im Transformatoröl kein Wasserstoff festgestellt werden. Innerhalb einiger Monate wurden die Ausgangskonzentrationen von $\mathrm{O}_{2}$ und $\mathrm{N}_{2}$ im Öl wieder erreicht. Sowohl die durch die Entgasung als auch durch die Rücksättigung bedingten deutlichen Sauerstoffkonzentrationsänderungen beeinflussten die ermittelten Wasserstoffkonzentrationen nicht.

\section{Wasserstoffmessung mit einem optischen Sensor}

Die optischen und elektrischen Eigenschaften von Palladiumschichten können sich beim Kontakt mit Wasserstoff ändern. Andere Gase bewirken solche Effekte nicht. Es wurde ein Sensor mit einer nanoskaligen Palladiumclusterschicht (Entwicklungsmuster, Materion $\mathrm{GmbH}$ ) untersucht. Experimentell wurden keine Querempfindlichkeiten von Sauerstoff, Kohlenoxiden und Kohlenwasserstoffen (C1-C3) gefunden. Aus dem Vergleich der Messreihen in den Bildern 7 und 8 wird deutlich, dass dieser Sensor nicht die für den Einsatz in OnlineSystemen zum Transformatorgasmonitoring notwendige Langzeitstabilität besitzt.

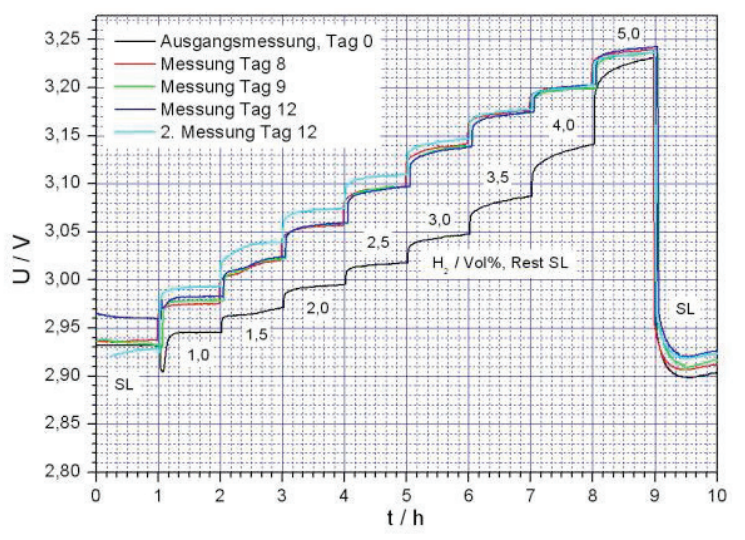

Bild 7 Stabilitätsmessungen eines optischen Wasserstoffsensors, Teil 1

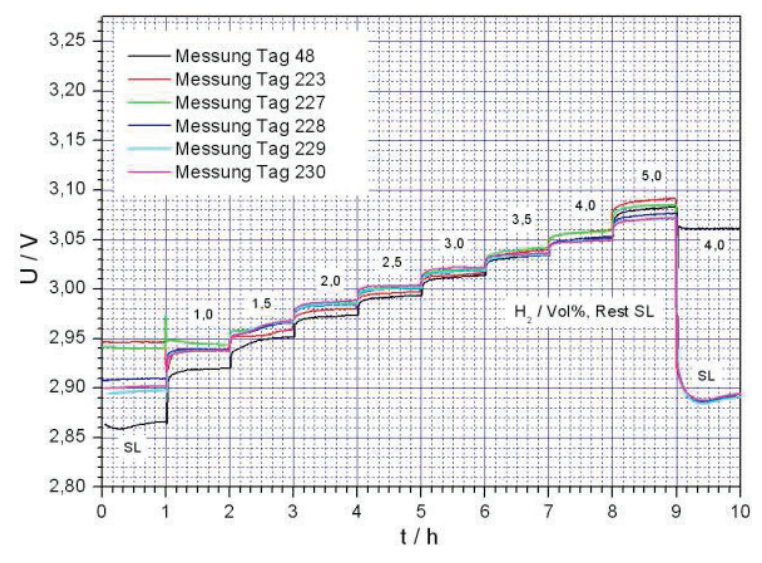

Bild 8 Stabilitätsmessungen eines optischen Wasserstoffsensors, Teil 2 


\section{Zusammenfassung}

Im Beitrag werden Untersuchungen zur Langzeitstabilität von Sensorkombinationen zur Wasserstoffmessung in Transformatorgasen, die in Gasmonitoren der Gatron $\mathrm{GmbH}$ über einen Zeitraum von bis zu zehn Jahren betrieben wurden, vorgestellt. Systematische Empfindlichkeitsänderungen der untersuchten Sensoren (WLD, paramagnetischer Sauerstoffsensor, NDIR-Sensoren) wurden nicht festgestellt. Die Empfindlichkeitsschwankungen lagen jeweils innerhalb der Messunsicherheiten des Verfahrens. Es konnte gezeigt werden, dass die beschriebene Methode zur Ermittlung der Wasserstoffkonzentration aus dem WLDSignal für den Einsatz in Online-Transformatorgas-Monitoren auch für lange Betriebszeiträume und stark schwankende Sauerstoffkonzentrationen im Öl geeignet ist. Ein Sensor auf Basis einer Palladiumclusterschicht erwies sich als sehr selektiv für Wasserstoff. Seine Empfindlichkeit nahm über einen Zeitraum von mehreren Monaten aber deutlich ab.

\section{Literatur}

[1] E DIN EN 60599 2013-12, In Betrieb befindliche, mit Mineralöl impregnierte elektrische Geräte Leitfaden zur Interpretation der Analyse gelöster und freier Gase (IEC 10/907CD:2013)

[2] DIN EN 60567:2011, Ölgefüllte elektrische Betriebsmittel - Probenahme von Gasen und Analyse freier und gelöster Gase - Anleitung

[3] CIGRE Technical Brochure 409, WG D1.01 (TF 15), Report on Gas Monitors for Oil-Filled Electrical Equipment, February 2010, ISBN 9782-85873-096-4

[4] T. Hübert, L. Boon-Brett, G. Black, U. Banach, Hydrogen Sensors - A review, Sensors and Actuators B 157 (2011) 329-352

[5] Jin-Seo Noh, Jun Min Lee, Wooyoung Lee, LowDimensional Palladium Nanostructures for Fast and Reliable Hydrogen Gas Detection, Sensors 2011, 11, 825-851; ISSN 1424-8220

[6] I. Pavlovsky, Hydrogen Sensor for Oil Transformer Health Monitoring, Nanotechnology, 2008, 211-213

[7] Landesanstalt für Umwelt, Messungen und Naturschutz Baden-Würtemberg, http://www.lubw.badenwuertemberg.de/servlet/is/18340, (30.09.2015)

[8] DIN EN 60422:2013-11, Isolieröle auf Mineralölbasis in elektrischen Betriebsmitteln Leitlinie zur Überwachung und Wartung

[9] E. Bräsel, Beiträge zur gasanalytischen Diagnostik von Öltransformatoren mit Luftabschlusssystem, Dissertation, Greifswald 1981
Teile dieser Arbeit wurden mit Mitteln des "Europäischen Sozialfonds" (ESF) gefördert. 